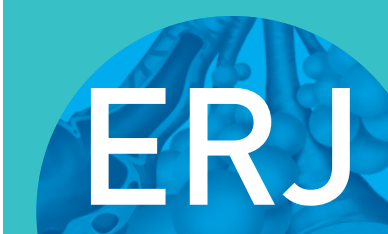

open research

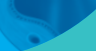

\title{
Predictors of non-cystic fibrosis bronchiectasis in Indigenous adult residents of central Australia: results of a case-control study
}

\author{
Lloyd Einsiedel (1) ${ }^{1}$, Hai Pham¹, Virginia Au², Saba Hatami², Kim Wilson³, \\ Tim Spelman ${ }^{4}$ and Hubertus Jersmann ${ }^{5}$
}

Affiliations: ${ }^{1}$ Baker Heart and Diabetes Institute, Alice Springs, Australia. ${ }^{2}$ Flinders Medical Centre, Adelaide, Australia. ${ }^{3}$ National Serology Reference Laboratory, Melbourne, Australia. ${ }^{4}$ Burnet Institute, Melbourne, Australia. ${ }^{5}$ Dept of Respiratory Medicine, Royal Adelaide Hospital, Adelaide, Australia.

Correspondence: Lloyd Einsiedel, Baker Heart and Diabetes Institute Central Australia, Rubuntja Building, Alice Springs Hospital, Gap Road, Alice Springs, Northern Territory 0870, Australia.

E-mail: lloyd.einsiedelant.gov.au

ABSTRACT The human T-cell leukaemia virus type 1 (HTLV-1) is associated with pulmonary inflammation. Indigenous Australians in central Australia have a very high prevalence of HTLV-1 infection and we hypothesised that this might contribute to high rates of bronchiectasis in this population.

80 Indigenous adults with confirmed bronchiectasis, each matched by age, sex and language to two controls without bronchiectasis, were recruited. Case notes and chest imaging were reviewed, HTLV-1 serology and the number of peripheral blood leukocytes (PBLs) infected with HTLV-1 (pro-viral load $(\mathrm{PVL})$ ) were determined, and radiological abnormality scores were calculated. Participants were followed for a mean \pm SD of $1.14 \pm 0.86$ years and causes of death were determined.

Median (interquartile range) HTLV-1 PVL for cases was 8-fold higher than controls (cases 213.8 (19.7-3776.3) copies per $10^{5}$ PBLs versus controls 26.6 (0.9-361) copies per $10^{5}$ PBLs; $\mathrm{p}=0.002$ ). Radiological abnormality scores were higher for cases with HTLV-1 PVL $\geqslant 1000$ copies per $10^{5}$ PBLs and no cause of bronchiectasis other than HTLV-1 infection. Major predictors of bronchiectasis were prior severe lower respiratory tract infection (adjusted OR (aOR) 17.83, 95\% CI 4.51-70.49; $\mathrm{p}<0.001$ ) and an HTLV-1 PVL $\geqslant 1000$ copies per $10^{5}$ PBLs (aOR 12.41, 95\% CI 3.84-40.15; p<0.001). Bronchiectasis (aOR 4.27, 95\% CI 2.04-8.94; $\mathrm{p}<0.001$ ) and HTLV-1 PVL $\geqslant 1000$ copies per $10^{5}$ PBLs (aOR 3.69, 95\% CI $1.11-12.27 ; \mathrm{p}=0.033)$ predicted death.

High HTLV-1 PVLs are associated with bronchiectasis and with more extensive radiological abnormalities, which may result from HTLV-1-mediated airway inflammation.

@ERSpublications

Higher numbers of HTLV-1-infected cells in peripheral blood are associated with bronchiectasis and more extensive radiological abnormalities among those with no cause for bronchiectasis other than HTLV-1 infection http://bit.ly/2V6pw98

Cite this article as: Einsiedel L, Pham $\mathrm{H}, \mathrm{Au} \mathrm{V}$, et al. Predictors of non-cystic fibrosis bronchiectasis in Indigenous adult residents of central Australia: results of a case-control study. ERJ Open Res 2019; 5: 00001-2019 [https://doi.org/10.1183/23120541.00001-2019].

This article has supplementary material available from openres.ersjournals.com

Received: 04 Jan 2019 | Accepted after revision: 14 Sept 2019

Copyright $\odot E R S$ 2019. This article is open access and distributed under the terms of the Creative Commons Attribution Non-Commercial Licence 4.0. 


\section{Introduction}

Bronchiectasis is characterised by the pathological dilatation of bronchi, which is thought to result from cycles of infection and inflammation in individuals with a dysregulated immune response [1,2]. Marked differences exist between populations in their prevalence of non-cystic fibrosis (non-CF) bronchiectasis. For example, prevalence in non-Indigenous populations of high-income countries is highest for females and the elderly [3,4]. Indigenous people of such countries are disproportionately affected [5], and disease occurs at a younger age $[6,7]$, more often involves males $[5,8]$ and outcomes are worse than for their non-Indigenous peers $[5,8]$. In central Australia, for example, $>1.0 \%$ of Indigenous adults were admitted with complications of bronchiectasis over 7 years and $34 \%$ died during 7.5 years at a mean age of only 42.5 years [5].

The reason for such a high prevalence of bronchiectasis in the Indigenous population of central Australia is unclear. Tuberculosis, measles and pertussis are important causes of bronchiectasis in low- and middle-income countries, but are uncommon in central Australia. Other recognised causes, such as immunoglobulin deficiency, are rare $[9,10]$ and CF has not been reported $[9,10]$. Childhood non-CF bronchiectasis has therefore been attributed to recurrent pneumonia resulting from overcrowded housing and barriers to personal hygiene [10]. The adult prevalence of infection with the human T-cell leukaemia virus type 1 (HTLV-1) exceeds $40 \%$ in some central Australian communities [11] and this may contribute to high rates of adult non-CF bronchiectasis $[5,12]$.

HTLV-1 is a human retrovirus that infects at least $5-10$ million people worldwide [13]. The virus is transmitted by exposure to infected lymphocytes in breast milk and blood, and through sexual intercourse [14]. HTLV-1-associated diseases are thought to arise in up to $10 \%$ of people with HTLV-1 [14]. Recognised complications include adult T-cell leukaemia/lymphoma (ATL), inflammatory disorders (HTLV-1-associated myelopathy (HAM) [15], alveolitis [16] and uveitis [14]) and severe infections with parasites, such as Strongyloides stercoralis [14]. Risk of disease increases markedly among people with higher numbers of HTLV-1-infected cells in peripheral blood (HTLV-1 pro-viral load (PVL)) [17]. The HTLV PVL varies as much as 1000 -fold between individuals, but is relatively stable over time within an individual [18].

Cross-sectional studies suggest that HTLV-1-associated pulmonary inflammation involves the alveoli, bronchi and bronchioles [16, 19-23]. Case series from several countries also suggest an association with bronchiectasis [22-24]; however, this is supported by a single small case-control study that did not control for post-infective bronchiectasis [12]. We now report the results of a larger case-control study that addresses these limitations and defines the risk factors for non-CF bronchiectasis in an adult Indigenous Australian population.

\section{Methods}

Study setting

A single, 186-bed hospital, Alice Springs Hospital (ASH), serves central Australia, a region of $\sim 1000000 \mathrm{~km}^{2}$ in which most Indigenous people live in conditions of considerable socioeconomic disadvantage. Patients requiring bronchoscopy are flown to tertiary referral centres $1500 \mathrm{~km}$ away.

\section{Recruitment and case-control matching}

The study aimed to prospectively recruit all Indigenous adults (aged $\geqslant 18$ years) admitted to ASH with a diagnosis of bronchiectasis (June 1, 2010 to December 31, 2013). Potential cases were identified by the project coordinator $(\mathrm{H}$. Pham) from the patient management system, and these were eligible for recruitment if 1) admitted to ASH for $>48 \mathrm{~h}$, and 2) the diagnosis of bronchiectasis met American College of Chest Physicians criteria [25]. Admission for $>48 \mathrm{~h}$ was required to ensure recruitment in primary languages by Aboriginal research team members, unaware of HTLV-1 serostatus, who worked standard office hours. Among 120 adults admitted with a diagnosis of bronchiectasis in the time period, 106 (94\%) were recruited. Chest high-resolution computed tomography (HRCT) for 104 potential cases was independently reviewed by two consultant radiologists (V. Au and S. Hatami), blind to HTLV-1 status, using the Picture Archiving and Communication System with InteleViewer software (www.intelerad.com). 26 participants were excluded because radiological criteria for bronchiectasis were not met (figure 1). Chest HRCT was not available for two cases: bronchiectasis was confirmed by chest radiography findings of cystic bronchiectasis $(\mathrm{n}=1)$ and bronchography in childhood $(\mathrm{n}=1)$.

Controls were Indigenous adults admitted to surgical or medical wards during the same period with 1) no lower respiratory tract infection (LRTI), 2) no evidence of bronchiectasis on chest radiography during the study period (no chest radiography: $\mathrm{n}=15$ controls) and 3) no discharge diagnosis of bronchiectasis or HTLV-1-associated diseases (ATL, HAM, uveitis or infective dermatitis) to end of follow-up [14]. In central Australia, HTLV-1 seroprevalence varies with age, sex and place of origin [26]. The latter is closely 


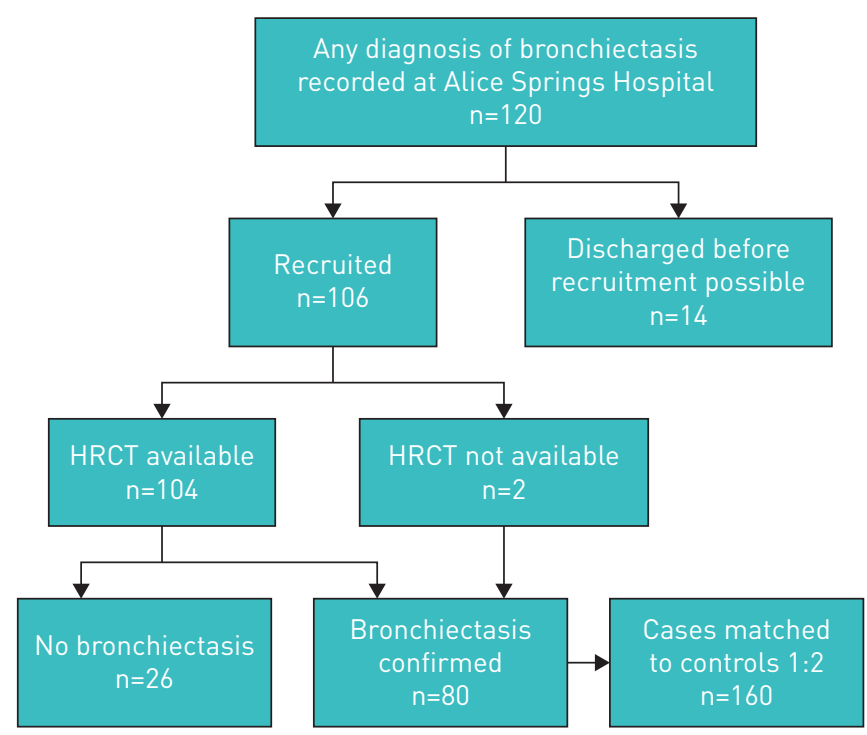

FIGURE 1 Recruitment flowchart for cases and matching to controls: recruitment was based on discharge diagnosis. Among 106 subjects with a discharge diagnosis of bronchiectasis, 104 were examined by chest high-resolution computed tomography (HRCT), which confirmed the diagnosis in 78 cases. Chest HRCT was not available for two cases for which diagnosis was confirmed by chest radiography findings of cystic bronchiectasis and by bronchography. Each case was then matched to two controls who were admitted during the study period with 1) no lower respiratory tract infection on admission, 2) no evidence of chronic lung disease on chest radiography and 3) no discharge diagnosis of either bronchiectasis or a human T-cell leukaemia virus type 1-associated disease. Reasons for admission of controls included: 1) surgical management $n=121(75.6 \%)$ (skin and soft tissue infections $n=45$, orthopaedic $n=22$, general surgical $n=20$, trauma $n=27$, pancreatitis $n=3$, burns $n=3$, tonsillectomy $n=1)$, 2) medical reasons $n=36(22.5 \%)$ (heart disease $n=11$, renal disease $n=8$, neurological disease $n=6$, gastroenterological disorders $n=4$, diabetic ketoacidosis $n=1$, constipation $n=2$, pelvic inflammatory disease $n=1$, alcohol withdrawal $n=1$, malnutrition $n=1$, acute rheumatic fever $n=1)$ and 3 ) to care for other patients $n=3(1.9 \%)$.

related to primary Indigenous languages, which were used to match cases and controls due to population mobility. Controls were matched to cases in a 2:1 ratio, by age group (age of case \pm 10 years), sex and primary Indigenous language by the project coordinator ( $\mathrm{H}$. Pham) who was blinded to HTLV-1 status.

Reasons for admission, comorbid conditions and results of relevant investigations were extracted from case notes for each admission (first admission to recruitment date) using a standardised data collection form. Data are presented to date of diagnosis for cases unless otherwise stated because LRTI presentations increase after bronchiectasis develops. Data for controls are presented to date of recruitment. Cases and controls were followed-up by reviewing medical records. Causes of death were determined to October 31, 2016, from death certificates held at the registries of births, deaths and marriages, Northern Territory of Australia and South Australia. Survival of participants with no death certificate was confirmed by hospital or clinic attendance to August 12, 2018 (no recorded attendance: $\mathrm{n}=6$ controls).

\section{Blood tests}

All cases and controls were tested for HTLV-1 and S. stercoralis serology. Routine investigations to establish a cause of bronchiectasis included full blood examination, serum immunoglobulins ( $\operatorname{IgG}, \operatorname{IgA}$ and IgM), IgG subclasses, total IgE, Aspergillus specific IgE and IgG, antinuclear antibodies, extractable nuclear antibodies, rheumatoid factor, and $\alpha_{1}$-antitrypsin. Tests for CF are not routine for Indigenous adults at ASH because no case has been found despite extensive testing $[9,10]$.

\section{Radiological scoring}

A "radiological abnormality score" was calculated from chest HRCT [12]. Briefly, each lobe was scored independently for bronchiectasis severity (0-3), bronchial wall thickening (0-3), mucus plugging/ centrilobular nodules $(0,1)$, sacculation $(0,1)$, parenchymal changes of ground-glass density $(0,1)$ and air trapping/emphysema $(0,1)$. A maximum score of 10 could therefore be assigned to each lobe. This was also assigned to lobes with complete collapse/cicatrisation. The lingular segment of the left upper lobe was regarded as a separate lobe. 


\section{Clinical definitions}

LRTI required a clinical diagnosis in the case record. Pneumonia was an LRTI with consolidation on chest imaging. Severe pneumonia required any of 1) lobar consolidation in two or more lobes, 2) lung abscess/empyema or 3) intensive care unit admission. Severe bronchiolitis required oxygen therapy for $>48 \mathrm{~h}$. Severe LRTI included severe pneumonia, severe bronchiolitis and pulmonary tuberculosis. Chronic obstructive pulmonary disease (COPD) required a clinical diagnosis with radiological findings of hyperinflation and reduced lung markings. Baseline spirometry was generally unavailable because of low attendance rates for outpatient lung function tests. Post-infective bronchiectasis was diagnosed when symptom onset clearly followed a prior severe LRTI. Chronic respiratory failure was defined as a stable daytime arterial oxygen tension $\leqslant 55 \mathrm{mmHg}(\leqslant 7.3 \mathrm{kPa})$. Allergic bronchopulmonary aspergillosis (ABPA) was diagnosed according to AGARwal et al. [27]. Sputum yield was defined as the number of cultures from which a pathogen was isolated divided by the number of cultures performed at least 1 month apart. Eosinophilia was defined as an eosinophil count in peripheral blood $>0.7 \times 10^{9} \mathrm{~L}^{-1}$ for $>12$ months.

\section{HTLV-1 studies}

Peripheral blood buffy coats were prepared at ASH and stored at $-80^{\circ} \mathrm{C}$, batched and transferred to the National Serology Reference Laboratory (Melbourne, Australia), where analyses were performed blinded to the patient's clinical state. Samples were initially screened for HTLV-1 plasma antibodies on both the Serodia HTLV-I particle agglutination assay (Fujirebio, Tokyo, Japan) and Murex HTLV-I+II test kit (Murex Diagnostics, Dartford, UK). Repeatedly reactive specimens underwent confirmatory testing using a more specific supplemental test, i.e. Western blot assay (HTLV-I/II Blot2.4; MP Biomedicals Asia Pacific, Singapore). HTLV-1c PVL was determined by real-time PCR as previously described [28]. The number of HTLV-1 copies per peripheral blood leukocyte (PBL) was expressed as HTLV-1 copies per $10^{5}$ PBLs. The lower limit of detection was 6.5 copies for HTLV-1 (95\% CI 5.4-8.4 copies) and 15.6 copies for albumin (95\% CI 12.9-20.0 copies), used as the internal control and to enumerate nucleated cells.

\section{Ethics statement}

The study was approved by the Central Australian Human Research Ethics Committee (HREC 14-202). All participants provided written consent.

\section{Statistical analysis}

Categorical variables were summarised using frequency (percentage). Continuous variables were summarised using mean with standard deviation or median (interquartile range (IQR)) as appropriate. Follow-up of cases and controls was to October 31, 2016. Cases and controls were compared using a Chi-squared, Fisher's exact or Wilcoxon rank-sum test as appropriate. Logistic regression was used to examine associations between bronchiectasis and demographic characteristics, clinical history and laboratory markers for all cases and their controls and for the subgroup with HTLV-1. Predictors of death from any cause among cases were also studied using logistic regression. The Hosmer-Lemeshow test was used to assess each model for goodness of fit. For all analyses $\mathrm{p}<0.05$ was considered significant. All analyses were conducted using Stata version 15 (StataCorp, College Station, TX, USA).

\section{Results}

Demographic and clinical characteristics of patients are shown in table 1. Reasons for admission of controls are listed in figure 1. Bronchiectasis was first diagnosed in childhood in $13(16.3 \%)$ cases at a mean \pm SD age of $5.3 \pm 4.1$ years; 67 cases $(83.7 \%)$ were diagnosed as adults (age $\geqslant 18$ years) at a mean \pm SD age of $43.1 \pm 15.4$ years. Males were most often affected (60\%) (table 1). High rates of smoking were recorded for both groups (table 1).

\section{Clinical details of bronchiectasis cases}

Bronchiectasis followed severe LRTI in 21 cases: pneumonia in 16 cases ( $n=9$ adults and $n=7$ children), bronchiolitis in four children and pulmonary tuberculosis in one adult. Other pathogens included Streptococcus pneumoniae $(\mathrm{n}=3)$, adenovirus $(\mathrm{n}=2)$, methicillin-susceptible Staphylococcus aureus $(\mathrm{n}=1)$ and Klebsiella pneumoniae $(\mathrm{n}=1)$. LRTIs were complicated by persistent lobar collapse in eight cases. Chronic lobar collapse was also documented prior to diagnosis for five cases with no identifiable antecedent event. 11 out of $13(84.6 \%)$ cases diagnosed with bronchiectasis in childhood, but only nine out of 67 (13.4\%) cases diagnosed in adulthood, were preceded by a severe LRTI (table 1).

Bronchiectasis was associated with combined IgA and IgG1-3 deficiency in one HTLV-1 uninfected case. Four cases had positive Aspergillus specific IgE with total $\mathrm{IgE}>1000 \mathrm{IU} \cdot \mathrm{mL}^{-1}$, but none met other criteria 


\section{TABLE 1 Demographic and clinical characteristics of cases and controls}

\begin{tabular}{|c|c|c|c|}
\hline & Cases & Controls & p-value \\
\hline Subjects & 80 & 160 & \\
\hline \multicolumn{4}{|l|}{ Demographics } \\
\hline Male & $48(60.0)$ & $96(60.0)$ & 1.000 \\
\hline Remote residence childhood & 53 (93.0) & 82 (85.4) & 0.237 \\
\hline Remote residence adulthood & $48(60.0)$ & 99 (61.9) & 0.449 \\
\hline Tobacco \# & $51(63.8)$ & 114 (71.3) & 0.237 \\
\hline Alcohol" & 54 (67.5) & $103(64.4)$ & 0.631 \\
\hline \multicolumn{4}{|l|}{ Comorbidities } \\
\hline $\mathrm{COPD}^{+}$ & $8(10.0)$ & $6(3.8)$ & 0.051 \\
\hline Asthma ${ }^{\S}$ & $1(1.3)$ & $0(0.0)$ & 0.333 \\
\hline Heart disease & $19(23.8)$ & $38(23.8)$ & 1.000 \\
\hline $\mathrm{CCF}$ & $7(8.8)$ & $11(6.9)$ & 0.603 \\
\hline IHD & $11(13.8)$ & $22(13.8)$ & 1.000 \\
\hline RHD & $1(1.3)$ & $5(3.1)$ & 0.380 \\
\hline Diabetes & $23(28.8)$ & 67 (41.9) & 0.048 \\
\hline Chronic kidney disease $^{f}$ & $11(13.8)$ & $38(23.8)$ & 0.161 \\
\hline Chronic liver disease & 9 (11.3) & $5(3.1)$ & 0.011 \\
\hline \multicolumn{4}{|l|}{ HTLV-1 infection } \\
\hline HTLV-1 infected $\# \#$ & 42 (52.5) & $53(33.1)$ & 0.004 \\
\hline HTLV-1 PVL copies per $10^{5}$ PBLs & $213.8(19.7-3776)$ & $26.6(0.9-361)$ & 0.002 \\
\hline \multicolumn{4}{|l|}{ Admission details ${ }^{7 \pi}$} \\
\hline \multicolumn{4}{|l|}{ Childhood admissions } \\
\hline Any admission ${ }^{++}$ & $55(68.8)$ & $72(45.0)$ & 0.035 \\
\hline Any LRTI admission ${ }^{\S \S}$ & $40(50.0)$ & 52 (32.5) & 0.009 \\
\hline ICU LRTI admissions & $0.04 \pm 0.19$ & $0.01 \pm 0.11$ & 0.317 \\
\hline LRTI admissions & $1(0-3)$ & $1(0-2)$ & 0.041 \\
\hline \multicolumn{4}{|l|}{ Adulthood admissions } \\
\hline Any admission ${ }^{++}$ & 49 (61.3) & 68 (42.5) & 0.005 \\
\hline Any LRTI admission $§ \S$ & 49 (61.3) & 67 (41.9) & 0.005 \\
\hline ICU LRTI admissions & $0.14 \pm 0.47$ & $0.06 \pm 0.33$ & 0.1166 \\
\hline LRTI admissions & $4(1-10)$ & $0(0-1)$ & $<0.001$ \\
\hline Severe LRTIff & $21(26.3)$ & $3(1.9)$ & $<0.001$ \\
\hline \multicolumn{4}{|l|}{ Mortality } \\
\hline Died & $31(38.8)$ & 18 (11.3) & $<0.001$ \\
\hline Age at death years & $50(36-59)$ & $58.5(44-63)$ & 0.0579 \\
\hline
\end{tabular}

Data are presented as $\mathrm{n}, \mathrm{n}(\%)$, median (interquartile range) or mean $\pm \mathrm{SD}$, unless otherwise stated. COPD: chronic obstructive pulmonary disease; CCF: congestive cardiac failure; IHD: ischaemic heart disease; RHD: rheumatic heart disease; HTLV-1: human T-cell leukaemia virus type 1; PVL: pro-viral load; PBL: peripheral blood leukocyte; LRTI: lower respiratory tract infection; ICU: intensive care unit. " : any history of tobacco smoking recorded in medical records; ": any history of harmful alcohol use recorded in medical records; ${ }^{+}$: history of COPD recorded in case notes with chest radiography findings consistent with this diagnosis (prior to bronchiectasis diagnosis for cases); ${ }^{\S}$ : clinical diagnosis of asthma recorded in case notes with an increased forced expiratory volume in $1 \mathrm{~s}>12 \%$ and $>200 \mathrm{~mL}$ after administration of bronchodilators; ${ }^{f}$ : stage $\geqslant 2$; ${ }^{\# \#}$ : HTLV-1 Western blot or HTLV-1C PCR positive; 1 "ी: prior to diagnosis for cases and to time of recruitment for controls; ${ }^{++}$: admission for any reason; ${ }^{\S}$ : admission for LRTI; ff: LRTI due to severe pneumonia, severe bronchiolitis or tuberculosis at any age (see Methods).

for ABPA. Although nontuberculous mycobacteria were more often cultured from the sputum of cases, these were not pathogens typically associated with pulmonary disease (supplementary table S1).

Cases were more likely to have HTLV-1 (42 out of 80 (52.5\%) versus controls 53 out of 160 (33.1\%); $\mathrm{p}=0.004$ ) and to have a higher HTLV-1 PVL (table 1). The median (IQR) HTLV-1 PVL for cases was 8 -fold higher than that of controls: cases 213.8 (19.7-3776) copies per $10^{5}$ PBLs versus controls 26.6 $(0.9-361)$ copies per $10^{5}$ PBLs ( $\left.\mathrm{p}=0.002\right)$ (table 1 ).

Radiological scores for bronchiectasis, bronchial wall thickening, saccular bronchiectasis, cystic bronchiectasis and overall radiological abnormality scores were significantly higher for cases with HTLV-1 PVL $\geqslant 1000$ copies per $10^{5}$ PBLs and no identifiable cause for bronchiectasis other than HTLV-1 infection (table 2). There was no difference in HTLV-1 PVL between cases with and without an alternative cause for bronchiectasis (figure 2). 
TABLE 2 Radiological abnormality scores for cases without alternative causes of bronchiectasis according to human T-cell leukaemia virus type 1 (HTLV-1) pro-viral load (PVL)

\begin{tabular}{|c|c|c|c|}
\hline & $\begin{array}{c}\text { Low PVL (<1000 copies per } \\
\left.10^{5} \mathrm{PBLs}\right)\end{array}$ & $\begin{array}{c}\text { High PVL }(\geqslant 1000 \text { copies per } \\
\left.10^{5} \text { PBLs }\right)\end{array}$ & p-value \\
\hline Subjects & 19 & 12 & \\
\hline Bronchiectasis score & $4.53 \pm 3.67$ & $7.25 \pm 3.86$ & 0.039 \\
\hline Bronchial wall thickening & $3.74 \pm 3.14$ & $6.17 \pm 3.01$ & 0.038 \\
\hline Cystic bronchiectasis & $0.68 \pm 1.25$ & $1.67 \pm 1.37$ & 0.028 \\
\hline Saccular bronchiectasis & $0.50 \pm 0.99$ & $1.47 \pm 1.55$ & 0.025 \\
\hline Ground-glass opacities & $0.85 \pm 0.96$ & $1.75 \pm 1.71$ & 0.144 \\
\hline $\begin{array}{l}\text { Mucus plugging/centrilobular } \\
\text { nodules }\end{array}$ & $1.21 \pm 1.47$ & $1.83 \pm 1.58$ & 0.248 \\
\hline $\begin{array}{l}\text { Total radiology abnormality } \\
\text { score }\end{array}$ & $11.68 \pm 8.56$ & $20.33 \pm 9.78$ & 0.022 \\
\hline
\end{tabular}

Data are presented as $\mathrm{n}$ or mean $\pm \mathrm{SD}$, unless otherwise stated. PBL: peripheral blood leukocyte. Combined radiological scores for all lobes for cases with HTLV-1 for which no alternative cause of bronchiectasis was found. Each lobe was scored separately by radiologists blinded to HTLV-1 status (see Methods). The lingular segment of the left upper lobe was regarded as a separate lobe. One case (HTLV-1 PVL 242 copies per $10^{5}$ PBLs) for which high-resolution computed tomography was not available for review was not included in this analysis. 10 cases with alternative causes were excluded (severe pneumonia $n=5$; empyema $n=2$; pulmonary abscess $n=1$; pulmonary tuberculosis $n=1$; severe childhood bronchiolitis $n=1$ ). \#: rank-sum test; १: a composite score comprising those for all other radiological parameters (bronchiectasis, bronchial wall thickening, cystic bronchiectasis, saccular bronchiectasis, mucus plugging/ centrilobular nodules and ground-glass opacities).

\section{Predictors of bronchiectasis}

The major predictors of bronchiectasis in a multivariable model were any admission with a severe LRTI (adjusted OR (aOR) 17.83, 95\% CI 4.51-70.49; $\mathrm{p}<0.001$ ) and an HTLV-1 PVL $\geqslant 1000$ copies per $10^{5}$ PBLs (aOR 12.41, 95\% CI 3.84-40.15; p<0.001) (table 3).

Among participants with HTLV-1, every 100 unit increase in HTLV-1 copies per $10^{5}$ PBLs was, on average, associated with 1.07 times the risk of bronchiectasis, which was 5.68-fold higher among participants with an HTLV-1 PVL $\geqslant 1000$ copies per $10^{5}$ PBLs in a multivariable model (table 4). Other

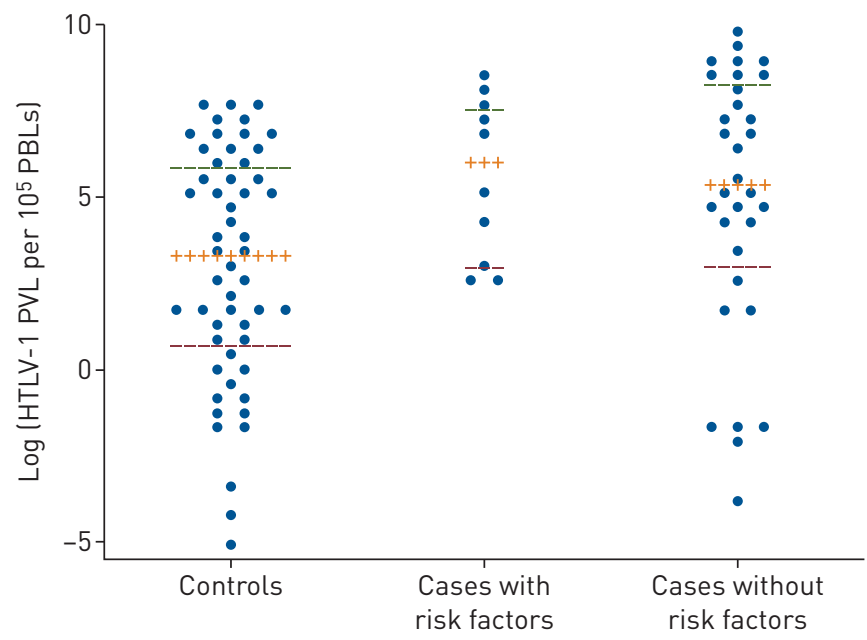

FIGURE 2 Dot plots with median (interquartile range (IQR)) comparing human T-cell leukaemia virus type 1 (HTLV-1) pro-viral load (PVL) for controls ( $n=53$ ), cases with HTLV-1 and risk factors for bronchiectasis ( $n=10$ ), and cases with no risk factors predisposing to bronchiectasis other than HTLV-1 infection ( $n=32)$. Risk factors for bronchiectasis included severe pneumonia $(n=5)$, empyema $(n=2)$, pulmonary abscess $(n=1)$, pulmonary tuberculosis ( $n=1)$ and severe childhood bronchiolitis ( $n=1)$. Median (IQR) HTLV-1 PVLs for controls, cases with other risk factors and cases without risk factors were 3.28 (0.49-5.89), 6.01 (2.98-7.58) and 5.35 (3.01-8.32) log unit copies per $10^{5}$ peripheral blood leukocytes (PBLs), respectively. HTLV-1 PVLs were significantly higher for cases with $(p=0.0178)$ and without $(p=0.0108)$ risk factors for bronchiectasis when compared with controls (Mann-Whitney test). There was no difference in HTLV-1 PVLs between groups for cases. 


\section{TABLE 3 Predictors of bronchiectasis among 80 cases and their 160 controls}

\begin{tabular}{|c|c|c|c|c|}
\hline & Unadjusted OR $(95 \% \mathrm{CI})$ & p-value & Adjusted OR (95\% CI) & p-value \\
\hline $\operatorname{Age}^{\#}$ & $1.01(0.99-1.03)$ & 0.355 & & \\
\hline Remote residence in adulthood" & $1.25(0.59-2.65)$ & 0.556 & & \\
\hline Admitted in childhood & $1.88(1.06-3.32)$ & 0.031 & & \\
\hline \multicolumn{5}{|l|}{ Any LRTI+ } \\
\hline Childhood & $2.08(1.20-3.60)$ & 0.009 & $1.90(0.93-3.86)$ & 0.078 \\
\hline Adulthood & $2.19(1.27-3.80)$ & 0.005 & & \\
\hline \multicolumn{5}{|l|}{ Number of LRTIs $s^{\S}$} \\
\hline Childhood & $1.15(0.95-1.40)$ & 0.151 & & \\
\hline Adulthood & $1.24(1.14-1.36)$ & $<0.001$ & & \\
\hline Number of ICU LRTIs ${ }^{f}$ & $1.28(0.56-2.95)$ & 0.558 & & \\
\hline Severe LRTI & $18.63(5.36-64.77)$ & $<0.001$ & $17.83(4.51-70.49)$ & $<0.001$ \\
\hline HTLV-1 infected & $2.23(1.29-3.86)$ & 0.004 & & \\
\hline \multicolumn{5}{|l|}{ HTLV-1 PVL (categorical) ${ }^{++}$} \\
\hline Uninfected & Reference & & Reference & \\
\hline Low HTLV-1 PVL & $1.50(0.81-2.76)$ & 0.194 & $1.92(0.88-4.19)$ & 0.101 \\
\hline High HTLV-1 PVL & $7.98(2.93-21.72)$ & $<0.001$ & $12.41(3.84-40.15)$ & $<0.001$ \\
\hline \multicolumn{5}{|l|}{ Sputum cultures } \\
\hline Yield ${ }^{\S \S}$ & $2.23(1.29-3.87)$ & 0.004 & $1.46(0.65-3.29)$ & 0.359 \\
\hline Haemophilus influenzae & $1.47(1.18-1.83)$ & 0.001 & $2.07(1.35-3.16)$ & 0.001 \\
\hline Streptococcus pneumoniae & $1.56(1.09-2.24)$ & 0.014 & $0.47(0.21-1.04)$ & 0.064 \\
\hline Pseudomonas aeruginosa & $2.79(0.76-10.32)$ & 0.124 & & \\
\hline NTM $^{f f}$ & $12.89(1.52-109.01)$ & 0.019 & $44.78(2.10-952.84)$ & 0.015 \\
\hline Eosinophilia\#\#\# & $2.88(1.65-5.03)$ & $<0.001$ & & \\
\hline Strongyloides ทีกา & $2.23(1.29-3.89)$ & 0.004 & $1.81(0.80-4.09)$ & 0.153 \\
\hline Cellulitis/skin abscess & $0.81(0.68-0.99)$ & 0.028 & $0.60(0.45-0.80)$ & 0.001 \\
\hline Infective dermatitis ${ }^{+++}$ & $2.70(1.28-5.70)$ & 0.009 & & \\
\hline Scabies $^{\S \S \S}$ & $6.06(1.93-19.02)$ & 0.002 & & \\
\hline Tobacco fff & $0.71(0.40-1.25)$ & 0.238 & & \\
\hline Alcohol \#\#\#\# & $1.15(0.65-2.03)$ & 0.631 & & \\
\hline
\end{tabular}

LRTI: lower respiratory tract infection; ICU: intensive care unit; HTLV-1: human T-cell leukaemia virus type 1; PVL: pro-viral load; NTM: nontuberculous mycobacteria. ${ }^{\#}$ : risk of bronchiectasis per 5 years. " : residence $>80 \mathrm{~km}$ from Alice Springs documented in medical records. ${ }^{+}$: admitted with any non-severe LRTI prior to diagnosis (cases) or date of recruitment (controls). §: number of admissions with LRTIs prior to diagnosis (cases) or date of recruitment (controls). ${ }^{f}$ : number of ICU admissions for LRTI in childhood and adulthood combined prior to diagnosis for cases or to date of recruitment for controls. \#\#: severe pneumonia $(n=16)$, severe bronchiolitis $(n=4)$ and pulmonary tuberculosis $(n=1)$ at any age (see Methods); includes one case with combined IgA and IgG deficiency; three controls had severe LRTI (pulmonary tuberculosis $n=1$, pulmonary abscess $n=1$, severe pneumonia $n=1$ ). ๆศी: HTLV-1 Western blot or HTLV-1c PCR positive. ${ }^{++}$: low HTLV-1 PVL, $<1000$ copies per $10^{5}$ peripheral blood leukocytes (PBLs); high HTLV-1 $\mathrm{PVL}, \geqslant 1000$ copies per $10^{5} \mathrm{PBLs}$. ${ }^{\S}$ : the number of pathogens isolated divided by the number of sputum samples collected, calculated once for each admission. ${ }^{f f}$ : NTM isolated from six cases prior to diagnosis included 1) a novel species that could not be identified together with a scotochromogenic mycobacterium, 2) Mycobacterium avium complex in a patient with pulmonary tuberculosis and 3) Mycobacterium simiae (isolated once from one case and twice from another); mycobacteria were not identified to species level in two cases and one control from which an NTM was isolated only once. ${ }^{\# \#}$ : eosinophilia recorded on at least two occasions 12 months apart (prior to diagnosis for cases). ๆกิา: Strongyloides seropositive or larvae identified in stool at any time prior to date of recruitment. ${ }^{+++}$: infective dermatitis recorded in case notes (cases HTLV $-1^{+} n=10, H T L V-1^{-} n=2$; controls HTLV $-1^{+} n=1, H T L V-1^{-} n=5$ ). ${ }^{\S \S}$; clinical diagnosis of scabies recorded in case notes (no case diagnosed by microscopy). fff: any history of tobacco smoking recorded in

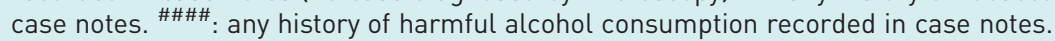

factors significantly associated with bronchiectasis in a multivariable model included a prior severe LRTI, a non-severe childhood LRTI and strongyloidiasis (table 4).

\section{Mortality}

During 272.9 years of follow-up after recruitment, 31 cases (38.8\%) and 18 controls (11.3\%) died at a median age of 50 and 58.5 years, respectively. Most cases died due to complications of bronchiectasis $(28$ out of $31(90.3 \%)$ ); the major cause of death for controls was cardiovascular disease (seven out of 18 (38.9\%)). The proportions of subjects who died among those without HTLV-1, those with an HTLV-1 


\begin{tabular}{|c|c|c|c|c|}
\hline & Unadjusted OR (95\% CI) & p-value & Adjusted OR $(95 \% \mathrm{CI})$ & p-value \\
\hline $\operatorname{Age}^{\#}$ & $1.01(0.88-1.16)$ & 0.870 & & \\
\hline Remote residence ${ }^{\pi}$ & $1.14(1.01-1.28)$ & 0.034 & $2.30(0.92-6.52)$ & 0.115 \\
\hline Admitted in childhood & $1.78(0.76-4.17)$ & 0.183 & & \\
\hline \multicolumn{5}{|l|}{ Any LRTI admission ${ }^{+}$} \\
\hline Childhood & $2.79(1.18-6.58)$ & 0.019 & $2.11(1.11-4.02)$ & 0.023 \\
\hline Adulthood & $3.02(1.28-7.14)$ & 0.012 & & \\
\hline \multicolumn{5}{|l|}{ Respiratory admissions ${ }^{\S}$} \\
\hline Childhood & $1.49(1.03-2.16)$ & 0.032 & & \\
\hline Adulthood & $1.41(1.17-1.71)$ & $<0.001$ & & \\
\hline Severe LRTI ${ }^{f}$ & $3.92(0.97-15.85)$ & 0.055 & $5.45(1.78-16.71)$ & 0.003 \\
\hline HTLV-1 PVL"\#\# & $1.07(1.02-1.12)$ & 0.006 & & \\
\hline \multicolumn{5}{|l|}{ HTLV-1 PVL (categorical) } \\
\hline Low HTLV-1 PVL & Reference & & Reference & \\
\hline High HTLV-1 PVL & $5.33(1.86-15.22)$ & 0.002 & 5.68 (1.81-17.89) & 0.003 \\
\hline \multicolumn{5}{|l|}{ Sputum cultures } \\
\hline Yield $^{++}$ & $4.24(1.55-11.60)$ & 0.005 & & \\
\hline Haemophilus influenzae & $1.67(1.16-2.42)$ & 0.006 & & \\
\hline Streptococcus pneumoniae & $3.56(1.36-9.29)$ & 0.010 & & \\
\hline Pseudomonas aeruginosa & $1.27(0.08-20.89)$ & 0.686 & & \\
\hline NTM S§ $^{\S}$ & $2.60(0.23-29.70)$ & 0.442 & & \\
\hline Eosinophiliaff $^{f}$ & $2.54(1.09-5.91)$ & 0.030 & & \\
\hline Strongyloides $\# \#$ & $2.53(1.40-4.58)$ & 0.002 & $2.44(1.22-4.89)$ & 0.012 \\
\hline Infective dermatitis & $3.76(1.15-12.34)$ & 0.029 & & \\
\hline Scabies $^{+++}$ & $7.23(1.49-35.09)$ & 0.014 & & \\
\hline Tobacco ${ }^{\S \S \S}$ & $0.94(0.40-2.24)$ & 0.897 & & \\
\hline Alcohol $^{f f f}$ & $1.79(0.72-4.42)$ & 0.208 & & \\
\hline
\end{tabular}

LRTI: lower respiratory tract infection; PVL: pro-viral load; NTM: nontuberculous mycobacteria. \#: risk of bronchiectasis per 5 years. " : residence $>80 \mathrm{~km}$ from Alice Springs in adulthood. ${ }^{+}$: admitted with any nonsevere LRTI prior to diagnosis (cases) or date of recruitment (controls). ${ }^{\S}$ : number of LRTI admissions prior to diagnosis (cases) or date of recruitment (controls). $f$ : severe pneumonia (n=8), severe bronchiolitis $(n=1)$, and pulmonary tuberculosis $(n=1)$ at any age (see Methods); three controls with HTLV-1 had a severe LRTI (pulmonary tuberculosis $n=1$, pulmonary abscess $n=1$, severe pneumonia $n=1$ ). \#\#: odds of bronchiectasis per 100 unit increase in HTLV-1 copies per $10^{5}$ peripheral blood leukocytes (PBLs). ๆा: low HTLV-1 PVL, $<1000$ copies per $10^{5}$ PBLs; high HTLV-1 PVL, $\geqslant 1000$ copies per $10^{5}$ PBLs. ${ }^{++}$: the number of pathogens isolated divided by the number of sputum samples collected, calculated once for each admission. $\S^{8}$ : NTM isolated from four cases prior to diagnosis included 1) Mycobacterium avium complex in a patient with pulmonary TB and 2) Mycobacterium simiae (isolated twice); NTM were not identified to species level in two cases from which an NTM was isolated only once. ${ }^{f f}$ : peripheral blood eosinophilia recorded on at least two occasions 12 months apart (prior to diagnosis for cases). \#\#\#: Strongyloides seropositive or larvae identified in stool at any time prior to date of recruitment (cases $n=18$ (Strongyloides seropositive $n=18$, larvae in stool $n=0$ ); controls $n=6$ (Strongyloides seropositive $n=5$, larvae in stool $n=1$ )). "ाาा?: infective dermatitis recorded in case notes (cases HTLV- $1^{+} n=10, H T L V-1^{-}$ $\mathrm{n}=2$; controls HTLV $-1^{+} \mathrm{n}=1, \mathrm{HTLV}-1^{-} \mathrm{n}=5$ ). ${ }^{++}$: clinical diagnosis of scabies recorded in case notes (no case diagnosed by microscopy). ${ }^{\S \S}$ : any history of tobacco smoking recorded in case notes. ${ }^{\mathrm{ff}}$ : any history of harmful alcohol consumption recorded in case notes.

PVL $<1000$ copies per $10^{5}$ PBLs and those with HTLV-1 PVL $\geqslant 1000$ copies per $10^{5}$ PBLs were 28 out of 145 (19.3\%), 11 out of $72(15.2 \%)$ and 10 out of $23(43.5 \%)$, respectively ( $p=0.031)$.

Univariable predictors of death included increasing age, bronchiectasis, COPD, HTLV-1 PVL $\geqslant 1000$ copies per $10^{5}$ PBLs, congestive cardiac failure and complications of bronchiectasis. In an adjusted model, bronchiectasis, COPD and HTLV-1 PVL $\geqslant 1000$ copies per $10^{5}$ PBLs remained predictive of death (table 5).

\section{Discussion}

Indigenous Australians have among the highest prevalence rates of adult non-CF bronchiectasis and the worst outcomes reported [5]. In the present study, the mean age of diagnosis was only 43 years and nearly $40 \%$ of cases died during the study period at a median age of 50 years. This reflects the 100 -fold higher prevalence of bronchiectasis among Indigenous adults relative to their non-Indigenous peers in central Australia [5] and the gap in life expectancy of 20 years between Indigenous and non-Indigenous adults 


\section{TABLE 5 Predictors of any death among cases and their controls}

\begin{tabular}{|c|c|c|c|c|}
\hline & Unadjusted OR (95\% CI) & p-value & Adjusted OR (95\% CI) & p-value \\
\hline Male & $1.61(0.82-3.15)$ & 0.169 & & \\
\hline HTLV-1 infected ${ }^{\#}$ & $1.00(0.52-1.90)$ & 1.000 & & \\
\hline \multicolumn{5}{|c|}{ HTLV-1 infection (categorical) } \\
\hline Uninfected & Reference & & Reference & \\
\hline Low HTLV-1 PVL & $0.75(0.35-1.62)$ & 0.467 & $2.27(0.93-5.54)$ & 0.071 \\
\hline High HTLV-1 PVL & $3.21(1.28-8.08)$ & 0.013 & $3.69(1.11-12.27)$ & 0.033 \\
\hline $\mathrm{Age}^{+}$ & $1.20(1.07-1.35)$ & 0.002 & $1.15(1.00-1.32)$ & 0.051 \\
\hline Remote residence ${ }^{\S}$ & $0.68(0.30-1.55)$ & 0.354 & & \\
\hline Bronchiectasis & $4.73(2.43-9.23)$ & $<0.001$ & $4.27(2.04-8.94)$ & $<0.001$ \\
\hline COPD $^{f}$ & $6.20(2.04-18.85)$ & 0.001 & $3.97(1.06-14.87)$ & 0.040 \\
\hline CCF & $2.81(1.03-7.69)$ & 0.044 & $2.27(0.73-7.03)$ & 0.155 \\
\hline IHD & $1.93(0.85-4.40)$ & 0.116 & & \\
\hline RHD & $0.80(0.09-6.97)$ & 0.837 & & \\
\hline Diabetes & $0.89(0.46-1.73)$ & 0.739 & & \\
\hline Haemodialysis $\# \#$ & $0.85(0.23-3.08)$ & 0.802 & & \\
\hline Chronic liver disease & $1.10(0.29-4.10)$ & 0.891 & & \\
\hline Malignancy & $2.74(0.44-16.87)$ & 0.277 & & \\
\hline Alcohol"17ก & $0.96(0.49-1.85)$ & 0.892 & & \\
\hline Tobacco $^{++}$ & $1.13(0.57-2.26)$ & 0.728 & & \\
\hline Eosinophilia ${ }^{\S \S}$ & $2.17(1.15-4.13)$ & 0.018 & & \\
\hline Strongyloides $f f$ & $1.36(0.68-2.72)$ & 0.380 & & \\
\hline \multicolumn{5}{|c|}{ Bronchiectasis complications } \\
\hline Respiratory failure & $12.01(4.55-31.71)$ & $<0.001$ & & \\
\hline Right heart failure & $5.73(1.24-26.52)$ & 0.026 & & \\
\hline Haemoptysis & $4.27(1.03-17.75)$ & 0.046 & & \\
\hline
\end{tabular}

HTLV-1: human T-cell leukaemia virus type 1; PVL: pro-viral load; COPD: chronic obstructive pulmonary disease; CCF: congestive cardiac failure; IHD: ischaemic heart disease; RHD: rheumatic heart disease. 31 cases and 18 controls died during follow-up. 28 cases, but no controls, died as a result of respiratory disease. Major causes of death among controls were cardiovascular disease $(n=7)$, malignancy $(n=3)$ and nonrespiratory sepsis $(n=3)$. " : HTLV-1 Western blot or HTLV-1c PCR positive; ๆ: stratified by HTLV-1 PVL (low HTLV-1 PVL, $<1000$ copies per $10^{5}$ peripheral blood leukocytes (PBLs); high HTLV-1 PVL, $\geqslant 1000$ copies per $10^{5} \mathrm{PBLs}_{;}{ }^{+}$: risk per 5 years; ${ }^{\S}$ : documented residence $>80 \mathrm{~km}$ from Alice Springs in adulthood; $f$ : diagnosis recorded in case notes with chest radiography findings consistent with diagnosis (cases diagnosed before bronchiectasis, controls to date of recruitment); ${ }^{\# \#}$ : six cases (HTLV- $1^{+}$ $\mathrm{n}=3$ ) and 12 controls (HTLV $-1^{+} \mathrm{n}=5$ ) were receiving haemodialysis at time of recruitment; " 1 : any history of harmful alcohol consumption recorded in case notes; ${ }^{++}$: any history of tobacco smoking recorded in case notes; $\S \S$ : peripheral blood eosinophilia recorded on at least two occasions 12 months apart (prior to diagnosis for cases); ${ }^{f f}$ : Strongyloides seropositive or larvae identified in stool at any time prior to date of recruitment.

with this condition [8]. Our finding that prior admissions with severe LRTI and high HTLV-1 PVL predicted bronchiectasis may contribute to this disparity; HTLV-1 infection is rare in non-Indigenous Australians [29]. A high HTLV-1 PVL was also associated with more extensive radiological abnormalities among cases for which HTLV-1 was the only identifiable cause of bronchiectasis and was an independent predictor of death during follow-up. These observations have not been reported previously.

Bronchiectasis is the end result of a "vicious cycle" of inflammation and infection caused by heterogeneous conditions that dysregulate the inflammatory response of susceptible individuals $[1,2]$. Vaccination against childhood respiratory infections, improved treatment of childhood bacterial infections and control of tuberculosis has led to significant changes in the epidemiology of bronchiectasis in high-income countries $[1,2]$. However, LRTI continues to be the most frequently cited of the identifiable aetiological factors in diverse countries, including Turkey [30, 31], the UK [32, 33] and China [34]. Similarly, post-infective bronchiectasis due to severe LRTI was the major identified cause of bronchiectasis in our socially disadvantaged study population, increasing risk 18 -fold in an adjusted model. Nevertheless, an antecedent severe LRTI could be identified in only nine out of 67 cases diagnosed in adulthood.

HTLV-1 is recognised to have a tropism for the pulmonary parenchyma [16]. Pulmonary involvement is associated with infiltration of HTLV-1-infected lymphocytes $[35,36]$ and an inflammatory cytokine milieu in bronchoalveolar lavage fluid [37]. In large Japanese case series, chest HRCT was abnormal in 30-61\% of adults with HTLV-1, including bronchiectasis in $15.6-22.5 \%$ of cases and bronchitis/bronchiolitis in 
23.6-29.5\% of cases [22, 23]. Both conditions are more common in Indigenous Australians with HTLV-1 [28]. The pathological correlate of these clinical observations is lymphocyte infiltration into bronchiole walls [22]. Functional alterations following HTLV-1 infection of CCR $4^{+} \mathrm{CD} 4^{+} \mathrm{T}$-cells lead to interferon- $\gamma$ production and a putative pro-inflammatory feedback loop in HAM [38]. Infiltration of HTLV-1-infected lymphocytes into the airways may similarly result in persistent inflammation and irreversible bronchial wall injury.

Inflammatory diseases resulting from HTLV-1 infection are associated with higher HTLV-1 PVL [17]. Median HTLV-1 PVLs for adults with HAM are 7-16-fold greater than those of asymptomatic adults [15, 39], and risk increases markedly when HTLV-1 PVL exceeds 1000 copies per $10^{5}$ peripheral blood mononuclear cells [15]. In the present study, the median HTLV-1 PVL was 8-fold higher for cases, and an HTLV-1 PVL $\geqslant 1000$ copies per $10^{5}$ PBLs was associated with higher bronchiectasis scores and more extensive radiological abnormalities on chest HRCT among cases for which HTLV-1-associated pulmonary disease was the only identifiable cause of bronchiectasis. An association between high HTLV-1 PVL and radiological abnormalities was previously reported in a study that combined chest HRCT data for subjects with bronchiectasis and chronic bronchitis [12]. Although HTLV-1 PVLs prior to presentation with bronchiectasis are unknown in the present study, our findings are consistent with an HTLV-1-mediated inflammatory process driven by high HTLV-1 PVL. High rates of bronchiectasis among otherwise asymptomatic Japanese adults with HTLV-1 [22, 23], and associations between HAM and bronchiectasis in the UK [40] and Brazil [24], suggest that this effect is not restricted to Indigenous Australians.

Strengths of the present study include the recruitment of nearly all eligible patients, the blinding to HTLV-1 status of researchers who recruited subjects and radiologists who independently reviewed imaging, and the blinding of laboratory staff to the clinical status of subjects. Moreover, all residents with a significant LRTI are admitted to ASH, which is the only hospital serving central Australia. Sputum mycobacterial cultures and standard blood tests for conditions associated with bronchiectasis were performed for nearly all cases $(75$ out of $80(93.8 \%))$. Nevertheless, limitations to the study design must be recognised. First, controls were not screened by chest HRCT. Individuals with early bronchiectasis may therefore have been included and our results may not be applicable to less severe bronchiectasis managed in the community. Second, all information was collected retrospectively and the diagnosis of some clinical diagnoses, such as scabies, could not be confirmed. The interpretation of LRTI data for cases prior to diagnosis with bronchiectasis is also limited because lengthy delays in diagnosis are common [41] and hospital referral with less severe LRTI may be more likely for individuals with frequent LRTI presentations.

In conclusion, two major risk factors for non-CF bronchiectasis were identified for Indigenous adults in central Australia. A severe LRTI, frequently complicated by persistent lobar collapse, was the major antecedent condition. Pulmonary outcomes for such patients could be improved by routine respiratory physician follow-up. High HTLV-1 PVL was also associated with bronchiectasis and more extensive radiological abnormalities, including higher bronchiectasis scores, among cases for which HTLV-1 was the only identifiable cause of bronchiectasis. Notwithstanding high rates of HTLV-1 infection in central Australia [11], there is currently no coordinated public health strategy to reduce HTLV-1 transmission among Indigenous Australians.

Acknowledgements: We gratefully acknowledge the work of Magdeline Lynch, Clinton Pepperill and Lena McCormack (Baker Heart and Diabetes Institute, Alice Springs, Australia) who recruited participants to this study, and Radwan Talukder (Baker Heart and Diabetes Institute) who assisted with the statistical analysis, and the support of the Alice Springs Hospital pathology service.

Conflict of interest: None declared.

Support statement: This study received funding from the National Health and Medical Research Council of Australia (NHMRC project grant 1012945). Funding information for this article has been deposited with the Crossref Funder Registry.

\section{References}

1 McShane P, Naureckas E, Tino G, et al. Non-cystic fibrosis bronchiectasis. Am J Respir Crit Care Med 2013; 188: 647-656.

2 Boyton R, Altmann D. Bronchiectasis: current concepts in pathogenesis, immunology, and microbiology. Annu Rev Pathol Mech Dis 2016; 11: 523-554.

3 Seitz A, Olivier K, Adjemian J, et al. Trends in bronchiectasis among Medicare beneficiaries in the United States, 2000 to 2007. Chest 2012; 142: 432-439.

4 Quint J, Millett E, Joshi M, et al. Changes in the incidence, prevalence and mortality of bronchiectasis in the UK from 2004 to 2013: a population-based cohort study. Eur Respir J 2016; 47: 186-193.

5 Einsiedel L, Fernandes L, Spelman T, et al. Bronchiectasis is associated with human T-lymphotropic virus 1 infection in an Indigenous Australian population. Clin Infect Dis 2012; 54: 43-50. 
6 Singleton R, Morris A, Redding G, et al. Bronchiectasis in Alaska native children: causes and clinical courses. Pediatr Pulmonol 2000; 29: 182-187.

7 Chang AB, Masel JP, Boyce NC, et al. Non-CF bronchiectasis: clinical and HRCT evaluation. Pediatr Pulmonol 2003; 35: 477-483.

8 Blackall S, Hong J, King P, et al. Bronchiectasis in indigenous and non-indigenous residents of Australia and New Zealand. Respirology 2018; 23: 743-749.

9 Maxwell GM. Chronic chest disease in Australian Aboriginal children. Arch Dis Childhood 1972; 47: 897-901.

10 Valery PC, Torzillo PJ, Mulholland K, et al. Hospital-based case-control study of bronchiectasis in Indigenous children in Central Australia. Pediatr Infect Dis J 2004; 23: 902-908.

11 Einsiedel L, Pham H, Woodman R, et al. The prevalence and clinical associations of HTLV-1 infection in a remote Indigenous community. Med J Aust 2016; 205: 305-309.

12 Einsiedel L, Cassar O, Goeman E, et al. Higher human T-lymphotropic virus type 1 subtype C proviral loads are associated with bronchiectasis in Indigenous Australians: results of a case-control study. Open Forum Infect Dis 2014; 1: ofu23.

13 Gessain A, Cassar O. Epidemiological aspects and world distribution of HTLV-1 infection. Front Microbiol 2012; 3: 388 .

14 Verdonck K, Gonzalez E, Van Dooren S, et al. Human T-lymphotropic virus 1: recent knowledge about an ancient infection. Lancet Infect Dis 2007; 7: 266-281.

15 Nagai M, Usuku K, Matsumoto W, et al. Analysis of HTLV-1 proviral load in 202 HAM/TSP patients and 243 asymptomatic HTLV-1 carriers: high proviral load strongly predisposes to HAM/TSP. J Neurovirol 1998; 4 ; 586-593.

16 Sugimoto M, Nakashima H, Watanabe S, et al. T-lymphocyte alveolitis in HTLV-1 associated myelopathy. Lancet 1987; 2: 1220.

17 Martin F, Taylor G, Jacobson S. Inflammatory manifestations of HTLV-1 and their therapeutic options. Expert Rev Clin Immunol 2014; 10: 1531-1546.

18 Kwaan N, Lee T, Chafets DM, et al. Long-term variations in human T lymphotropic virus (HTLV)-I and HTLV-II proviral loads and association with clinical data. J Infect Dis 2006; 194: 1557-1564.

19 Couderc LJ, Caubarrere I, Venet A, et al. Bronchoalveolar lymphocytosis in patients with tropical spastic paraparesis associated with human T-cell lymphotropic virus type I (HTLV-I). Ann Intern Med 1988; 109; $625-628$.

20 Setoguchi Y, Takahashi S, Nukiwa T, et al. Detection of human T-cell lymphotropic virus type-1 related antibodies in patients with lymphocytic interstitial pneumonia. Am Rev Respir Dis 1991; 144: 1361-1365.

21 Sugisaki K, Tsuda T, Kunamoto T, et al. Clinicopathologic characteristics of lungs of patients with human T-cell lymphotropic virus type 1 associated myelopathy. Am J Trop Med Hyg 1998; 58: 721-725.

22 Okada F, Ando Y, Yoshitake S, et al. Pulmonary CT findings in 320 carriers of human T-lymphotropic virus type 1 . Radiology 2006; 240: 559-564.

23 Yamashiro T, Kamiya H, Miyara T, et al. CT scans of the chest in carriers of human T-cell lymphotropic virus type 1: presence of interstitial pneumonia. Acad Radiol 2012; 19: 952-957.

24 Magno Falcão LF, Falcão ASC, Medeiros Sousa RC. CT chest and pulmonary functional changes in patients with HTLV-associated myelopathy in the Eastern Brazilian Amazon. PLoS One 2017; 12: e0186055.

25 Rosen MJ. Chronic cough due to bronchiectasis: ACCP evidence-based clinical practice guidelines. Chest 2006; 129: Suppl. 1, 122S-131S.

26 Einsiedel L, Woodman R, Flynn M, et al. Human T-lymphotropic virus type 1 infection in an Indigenous Australian population: epidemiological insights from a hospital-based cohort study. BMC Public Health 2016; 16 787.

27 Agarwal R, Chakrabarti A, Shah A, et al. Allergic bronchopulmonary aspergillosis: review of literature and proposal of new diagnostic and classification criteria. Clin Exp Allergy 2013; 43: 850-873.

28 Einsiedel L, Pham H, Wilson K, et al. Human T-lymphotropic virus type 1c subtype proviral loads, chronic lung disease and survival in a prospective cohort of Indigenous Australians. PLoS Negl Trop Dis 2018; 12: e0006281.

29 Polizzotto $\mathrm{M}$, Wood $\mathrm{E}$, Ingham $\mathrm{H}$, et al. Reducing the risk of transfusion-transmissible viral infection through blood donor selection: the Australian experience 2000 through 2006. Transfusion 2008; 48: 55-63.

30 Börekçi S, Musellim B. Aetiology of bronchiectasis: evaluation of 319 adult patients with bronchiectasis. Eur Respir $\mathrm{J}$ 2016; 48: Suppl. 60, PA670.

31 Habesoglu MA, Ugurlu AO, Eyuboglu FO. Clinical, radiologic, and functional evaluation of 304 patients with bronchiectasis. Ann Thorac Med 2011; 6: 131-136.

32 Shoemark A, Ozerovitch L, Wilson R. Aetiology in adult patients with bronchiectasis. Respir Med 2007; 101: 1163-1170.

33 Pasteur MC, Helliwell SM, Houghton SJ, et al. An investigation into causative factors in patients with bronchiectasis. Am J Crit Care Med 2000; 162: 1277-1284.

34 Qi Q, Wang W, Li T, et al. Aetiology and clinical characteristics of patients with bronchiectasis in a Chinese Han population: a prospective study. Respirology 2015; 20: 917-924

35 Mori S, Mizoguchi A, Kawabata M, et al. Bronchoalveolar lavage lymphocytes correlate with HTLV-1 proviral load in HTLV-1 carriers. Thorax 2005; 60: 138-143.

36 Kawabata T, Higashimoto I, Takashima H, et al. Human T-lymphotropic virus type I (HTLV-I)-specific CD8 ${ }^{+}$ cells accumulate in the lungs of patients infected with HTLV-I with pulmonary involvement. J Med Virol 2012; 84: $1120-1127$.

37 Yamazato Y, Miyazato A, Kawakami K, et al. High expression of $\mathrm{p} 40^{\text {tax }}$ and proinflammatory cytokines and chemokines in the lungs of human T-lymphotropic virus type-1-related bronchopulmonary disorder. Chest 2003 124: 2283-2292.

38 Yamano Y, Coler-Reilly A. HTLV-1 induces a Th1-like state in CD4 ${ }^{+} \mathrm{CCR}^{+} \mathrm{T}$ cells that produces an inflammatory positive feedback loop via astrocytes in HAM/TSP. J Neuroimmunol 2017; 304: 51-55.

39 dos Santos Brito Silva Furtado M, Andrade R, Romanelli L, et al. Monitoring the HTLV-1 proviral load in the peripheral blood of asymptomatic carriers and patients with HTLV-associated myelopathy/tropical spastic 
paraparesis from a Brazilian cohort: ROC curve analysis to establish the threshold for risk disease. J Med Virol 2012; 84: 664-671.

40 Honarbakhsh S, Taylor G. High prevalence of bronchiectasis is linked to HTLV-1-associated inflammatory disease. BMC Infect Dis 2015; 5: 258.

41 King PT, Holdsworth SR, Freezer NJ, et al. Characterisation of the onset and presenting features of adult bronchiectasis. Respir Med 2006; 100: 2183-2189. 